\title{
Side-by-Side Comparison of uPAR-Targeting Optical Imaging Antibodies and Antibody Fragments for Fluorescence-Guided Surgery of Solid Tumors
}

Victor M. Baart ${ }^{1}$, Labrinus van Manen ${ }^{1}$, Shadhvi S. Bhairosingh ${ }^{1}$, Floris A. Vuijk ${ }^{\text {, }}$ Luisa lamele ${ }^{2,3}$, Hugo de Jonge ${ }^{2,3}$, Claudia Scotti ${ }^{2,3}$, Massimo Resnati ${ }^{4}$, Robert A. Cordfunke ${ }^{5}$, Peter J. K. Kuppen ${ }^{1}$, Andrew P. Mazar ${ }^{6}$, Jacobus Burggraaf ${ }^{1,7}$, Alexander L. Vahrmeijer ${ }^{1}$, and Cornelis F. M. Sier ${ }^{1,8}$

${ }^{1}$ Department of Surgery, Leiden University Medical Center, Leiden, The Netherlands

${ }^{2}$ Unit of Immunology and General Pathology, Department of Molecular Medicine, University of Pavia, Pavia, Italy

${ }^{3}$ Ardis Srl, Pavia, Italy

${ }^{4}$ Age Related Diseases Unit, Division of Genetics and Cell Biology, San Raffaele Scientific Institute, Milano, Italy

${ }^{5}$ Department of Immunohematology and Blood Transfusion, Leiden University Medical Center, Leiden, The Netherlands

${ }^{6}$ Monopar Therapeutics, Wilmette, IL, USA

${ }^{7}$ Centre for Human Drug Research, Leiden, The Netherlands

${ }^{8}$ Percuros BV, Leiden, The Netherlands 2021

\begin{abstract}
Purpose: Radical resection is paramount for curative oncological surgery. Fluorescence-guided surgery (FGS) aids in intraoperative identification of tumor-positive resection margins. This study aims to assess the feasibility of urokinase plasminogen activator receptor (UPAR) targeting antibody fragments for FGS in a direct comparison with their parent IgG in various relevant in vivo models.

Procedures: Humanized anti-uPAR monoclonal antibody MNPR-101 (ulgG) was proteolytically digested into $F\left(a b^{\prime}\right) 2$ and Fab fragments named $u F a b 2$ and uFab. Surface plasmon resonance (SPR) and cell assays were used to determine in vitro binding before and after fluorescent labeling with IRDye800CW. Mice bearing subcutaneous HT-29 human colonic cancer cells were imaged serially for up to $120 \mathrm{~h}$ after fluorescent tracer administration. Imaging characteristics and ex vivo organ biodistribution were further compared in orthotopic pancreatic ductal adenocarcinoma (BxPc-3-luc2), head-and-neck squamous cell carcinoma (OSC-19-luc2-GFP), and peritoneal carcinomatosis (HT29-luc2) models using the clinical Artemis fluorescence imaging system.

Results: Unconjugated and conjugated ulgG, uFab2, and uFab specifically recognized uPAR in the nanomolar range as determined by SPR and cell assays. Subcutaneous tumors were clearly identifiable with tumor-to-background ratios (TBRs) $>2$ after $72 \mathrm{~h}$ for ulgG-800F and $24 \mathrm{~h}$ for uFab2-800F and $\mathrm{uFab}-800 \mathrm{~F}$. For the latter two, mean fluorescence intensities (MFIs) dipped below predetermined threshold after $72 \mathrm{~h}$ and $36 \mathrm{~h}$, respectively. Tumors were easily identified in the orthotopic models with ulgG-800F consistently having the highest MFIs and uFab2-800F and uFab-800F having similar values. In biodistribution studies, kidney and liver fluorescence approached tumor fluorescence after ulgG800F administration and surpassed tumor fluorescence after uFab2-800F or uFab-800F administration, resulting in interference in the abdominal orthotopic mouse models.
\end{abstract}

Correspondence to: Victor M. Baart; e-mail:V.M.Baart@lumc.nl 
Conclusions: In a side-by-side comparison, FGS with uPAR-targeting antibody fragments compared with the parent lgG resulted in earlier tumor visualization at the expense of peak fluorescence intensity.

Key words Image-guided surgery - Near infrared - Fluorescence - Urokinase plasminogen activator receptor $\cdot$ uPAR $\cdot \lg G \cdot F\left(a b^{\prime}\right) 2 \cdot$ Fab $\cdot$ Pancreatic cancer $\cdot$ Head and neck cancer . Oral cancer $\cdot$ Colon cancer $\cdot$ Fluorescence-guided surgery

\section{Introduction}

Cancer incidence is on the rise with one in five to six people awaiting a cancer diagnosis and one in eight to ten people eventually succumbing to the disease [1]. Despite recent advances in treatment, surgical resection remains the corner stone of curative therapy $[1,2]$. The primary aim of oncological surgery is to achieve local control by radical resection (i.e., R0, tumor-negative margins), as tumor-positive margins negatively influence disease-free and overall survival [3-5]. As a result, correctly discerning malignant from benign tissue during surgery is fundamental for resection.

Surgeons can utilize intraoperative imaging techniques to aid in this differentiation. A relatively novel technology, fluorescence-guided surgery (FGS), uses advanced camera systems to capture near-infrared (NIR) fluorescence emitted by contrast agents, targeting, for example, ureters, nerves, or tumors. FGS has the advantage that it is real-time, has a high contrast and sensitivity, does not utilize ionizing radiation, and is easy-to-use [6, 7]. For oncological FGS, the crux lies in developing fluorescent tracers that specifically target proteins which are present in malignant cells and absent or quiet in surrounding tissue. A promising target for imaging of disease, including various types of cancer, is the urokinase plasminogen activator receptor (UPAR) $[8,9]$.

UPAR is a three-domain cell membrane bound receptor that plays a pivotal role in growth factor activation and extracellular matrix remodeling, stimulating proliferation, differentiation, and migration of cells. Its presence in cancer correlates with prognostic outcome variables such as metastatic disease and reduced overall- and disease-free survival. Furthermore, immunohistochemical studies have revealed absent to low levels of UPAR expression in healthy homeostatic tissue whereas at the interface between tumor and healthy tissue, uPAR is highly overexpressed on both tumor and tumor-associated stromal cells [8-12]. Such a pattern is ideal for imaging and, not surprisingly, a PET tracer is currently undergoing extensive clinical trials in order to image "cancer aggressiveness" and subsequently aid in tailoring treatment options to disease biology [13].

As uPAR expression is highest at the tumor borders, our group has primarily focused on developing uPAR-targeted FGS tracers. We have successfully targeted uPAR for FGS in various in vivo human cancer models using a mouse monoclonal antibody and more recently with MNPR-101, a firstin-class humanized monoclonal antibody targeting domain three of UPAR [14-16]. This domain is ideal as recognition is independent of the often simultaneously upregulated uPARligands urokinase and vitronectin, and remains membrane bound when UPAR is cleaved [9].

The relatively large size of antibodies $(\approx 150$ kiloDalton $(\mathrm{kDa})$ ), however, might limit extravasation, and the interaction of their crystallizable fragment $(\mathrm{Fc})$ with the neonatal Fc receptor on endothelial cells results in an extended halflife, decreasing tissue penetration and delaying the optimal imaging window $[17,18]$. As a consequence, there is often a delay of 4 to 6 days after administration before patients can be imaged with optimal results [19]. Reducing molecular size, the dominant variable in determining extravasation (distribution) and clearance, shortens imaging times and improves tumor penetration [20]. Clinically, these advantages should result in improved tumor fluorescence and contrast at earlier time points resulting in better visualization for surgeons and more rapid surgery, also allowing surgeons to utilize fluorescence in (semi-) acute settings [21]. As a result, shortening the imaging window is often presented as the holy grail of FGS when introducing novel small-sized fluorescent tracers. Tumor specificity, tumor-to-background ratio (TBR), and tumor mean fluorescence intensity (MFI), however, are of equal importance. Positron emission tomography (PET) studies have for instance illustrated that antigen binding fragments $(\mathrm{Fab})$ and $\mathrm{F}\left(\mathrm{ab}^{\prime}\right)_{2}$ lead to much faster tumor targeting, but also a nearly two- to three-fold lower peak uptake compared to the parent antibody [22, 23]. Whether this is also true for FGS remains to be elucidated. The current study aims to determine how uPAR-targeting Fab and F(ab') $)_{2}$ compare to their parent full-sized antibody (MNPR-101) in FGS of orthotopic PDAC, HNSSC, and peritoneal carcinomatosis CRC models.

\section{Materials and Methods}

\section{Generation of Fab and $F\left(a b^{\prime}\right)_{2}$ from MNPR-101}

MNPR-101, a humanized antibody targeting uPAR, was enzymatically fragmented into Fab and $\left.\mathrm{F}(\mathrm{ab})_{2}\right)_{2}$ by respectively GingisKHAN and FabRICATOR kits, following the protocols of the manufacturer (Genovis AB, Lund, Sweden). The full-sized antibody and the generated fragments are further indicated as uIgG, uFab2, and uFab. Tracers (fullsized antibody and fragments) were conjugated with IRDye 
800CW-NHS ester (from here on 800F; LI-COR biotechnology, Lincoln, USA) according to the manufacturer and as published before [16]. Digestion and conjugation results were evaluated using SDS-PAGE on pre-casted 4-20\% gels (Criterion, Bio-Rad laboratories, Veenendaal, The Netherlands). Proteins on gels were stained with Coomassie brilliant blue G-250 (Bio-Rad laboratories, Veenendaal, The Netherlands) and fluorescence of $800 \mathrm{~F}$ was determined using an Odyssey imager (LI-COR biotechnology, Lincoln, USA). Degrees of labeling were calculated and verified by MALDI-TOF mass spectrometry as described previously [24].

\section{Surface Plasmon Resonance of Antibody Fragments}

All surface plasmon resonance (SPR) experiments were performed on a Biacore T200 (GE Healthcare Life Sciences, Uppsala, Sweden) using a NiHC1500M sensor chip (Xantec Bioanalytics, Düsseldorf, Germany) as described previously (see ESM for details) [16].

\section{Cells and Culture Conditions}

Culture conditions of human embryonic kidney empty vector (HEK EV), HEK uPAR wildtype (HEK uPAR WT), HEK uPAR D2-D3 isoform (HEK uPAR D2-3), OSC-19-luc2GFP, BxPC-3-luc2, and HT-29-luc2 cells are described in the ESM file.

\section{Flow Cytometry and Cell-Based Plate Assays}

For flow cytometry, cells were incubated in succession with primary antibody (-fragment), secondary anti-human IgG Fab fragment antibody (clone 4A11; ab771, Abcam, Cambridge, UK), and anti-mouse 488 antibody (A21121, Life Technologies, CA, USA) using a standard flow cytometry protocol and measured on a LSRII flow cytometer (BD Biosciences, CA, USA). Binding of serially diluted 800F-labeled antibodies to cells cultured in 96-well plates was determined using the Odyssey and fluorescence was corrected for cell density with a ToPro-3 iodide nuclear staining (T3605, Invitrogen, CA, USA). The CD52-targeting humanized antibody Alemtuzumab coupled to $800 \mathrm{~F}$ functioned as a conjugate control. For detailed methods, see ESM.

\section{In Vivo Tumor Models}

All in vivo experiments were approved by the Dutch Central Commission for Animal Experimentation (AVD1160020172925) and performed in accordance with the code of practice "Dierproeven In Het Kankeronderzoek". Six- to ten-week-old female BALB/c-Nude mice (CAnN.CgFoxnInu/Crl, Charles River Laboratories, MA, USA) were inoculated with tumor cells and randomized into experimental groups once tumors had reached predetermined sizes by digital caliper or bioluminescence using the IVIS ${ }^{\circledR}$ Spectrum Preclinical In Vivo Imaging System (Spectrum, PerkinElmer, MA, USA) (for details, see ESM). Subcutaneous tumors were induced by subcutaneous injection of 500,000 HT-29 cells. Orthotopic OSC-19-luc2-GFP HNSCC, BxPC-3-luc2 PDAC, and HT-29-luc2 CRC peritoneal carcinomatosis models were induced as previously published [15, 25, 26].

\section{Fluorescence Imaging Systems}

Fluorescence images were acquired using the preclinical Pearl Trilogy Small Animal Imaging System (Pearl; LICOR biotechnology, Lincoln, USA) and the Artemis clinical system (Quest Medical Imaging, Middenmeer, The Netherlands). Pearl images were analyzed with Image Studio 5.2 (LI-COR biotechnology, Lincoln, USA). Frames from Artemis recordings, taken with standardized exposure times, were captured with Spectrum Capture Suite 1.4.3 (Quest Medical Imaging, Middenmeer, The Netherlands) and analyzed with Fiji Image-J [27].

\section{Fluorescence Imaging}

One hundred microliters of fluorescent tracer was administered intravenously at equal number of antigen binding sites, respectively, $1 \mathrm{nmol}$ for $\mathrm{uIgG}-800 \mathrm{~F}$ and $\mathrm{uFab} 2-800 \mathrm{~F}$ and $2 \mathrm{nmol}$ for uFab-800F. Subcutaneous models were imaged at $1,2,4,12,24,36,48,72,96$, and $120 \mathrm{~h}$ while orthotopic models were imaged at the optimal imaging window after tumor exposure. Optimal time points were selected based on TBR and MFI cut-offs, literature, and visual interpretation. Outcome measures included TBR, MFI, and Artemis exposure times (see ESM for details).

\section{Post-mortem Histological Analysis}

Tumors were resected with surrounding normal tissue, embedded in paraffin, sectioned, and scanned for $800-\mathrm{nm}$ fluorescence using the Odyssey. After scanning, sections were stained for hematoxylin and eosin and digitalized with the Panoramic Digital slide Scanner and CaseViewer 2.3 (both 3D Histech, Budapest, Hungary). Overlays were created with Adobe Photoshop CC 2018 (Adobe Systems, CA, USA).

\section{Image Analysis and Statistics}

TBRs were calculated by dividing the tumor MFI with background MFI measured using the Pearl. For subcutaneous models, the background region-of-interest was the area next to the tumor equidistant from metabolizing organs. For the SSC, PDAC, and CRC peritoneal carcinomatosis models, the background region-of-interests were muscle, spleen, and peritoneum, respectively. Means were reported with standard deviations and compared using two-way ANOVA with 
GraphPad Prism 8 (GraphPad Software, CA, USA). A $p$ value $<0.05$ was considered statistically significant.

\section{Results}

\section{In Vitro Characterization of $u I g G, u F a b 2$, and $u F a b$}

MNPR-101 (150-155 kDa) was enzymatically digested and purified resulting in a $100-110 \mathrm{kDa}$ uFab2-800F and 50-55 kDa uFab (Fig. 1a). SPR determined the $\mathrm{K}_{\mathrm{D}}$ of $\mathrm{uIgG}, \mathrm{uFab} 2$, and uFab for recombinant uPAR to be $2.19 \times 10^{-10} \pm 2.41 \times 10^{-11} \mathrm{M}, 5.61 \times 10^{-10} \pm 1.61 \times 10^{-11} \mathrm{M}$, and $8.66 \times 10^{-10} \pm 4.17 \times 10^{-10} \mathrm{M}$, respectively (Table 1 ; for sensograms, see ESM file, Suppl. Figure 1). Affinities did not differ significantly from each other $(p=0.59) . K_{\mathrm{a}}$ and $K_{\mathrm{d}}$ values of all tracers can be found in Suppl. Table 1 (see ESM). Flow cytometry confirmed specificity for uPAR by showing a right shift in signal when $\mathrm{uIgG}, \mathrm{uFab} 2$, and $\mathrm{uFab}$ were incubated with HEK uPAR WT cells, HEK uPAR D2-3 cells, but not with HEK EV cells (Fig. 1b).

The calculated fluorescence labeling with $800 \mathrm{~F}$ was comparable for $\mathrm{uIgG}, \mathrm{uFab} 2$, and $\mathrm{uFab}$, and the $K_{\mathrm{D}}$ did not differ significantly after labeling (uIgG vs. uIgG-800F $p=0.47$; uFab2 vs. uFab2-800F $p=0.53$; uFab vs. uFab-800F $p=0.32$; Table 1). Cell-based plate assays showed a dose-dependent a

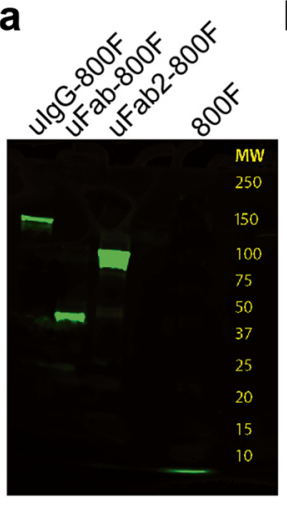

b

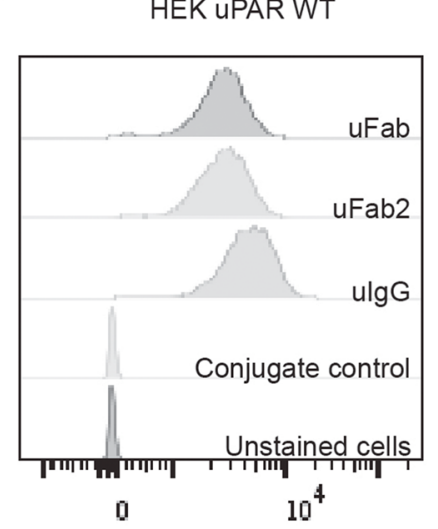

HEK UPAR D2-3

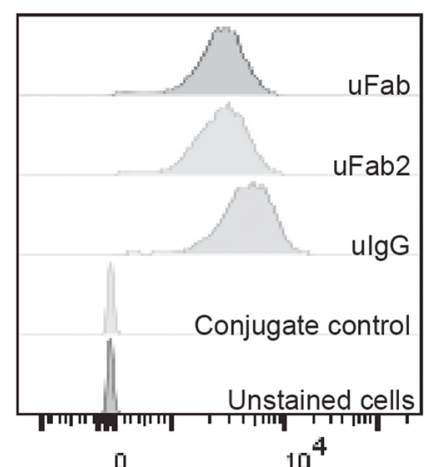

HEK UPAR D2-3
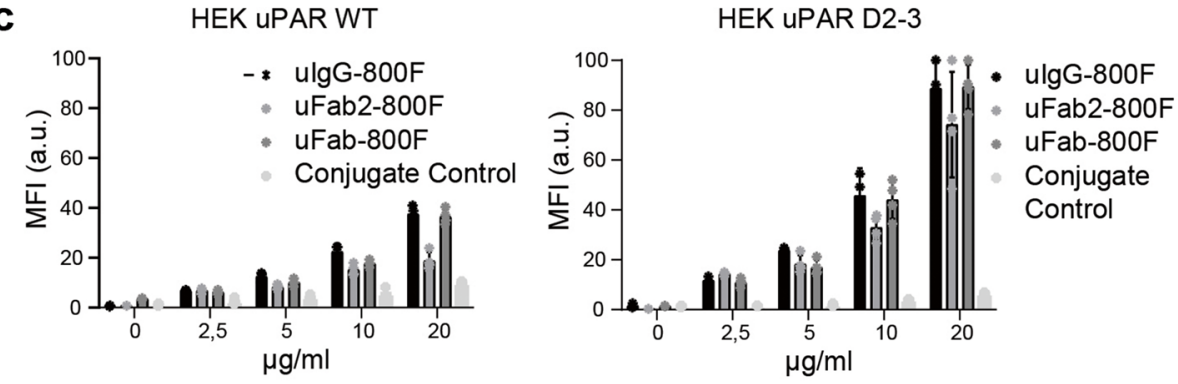

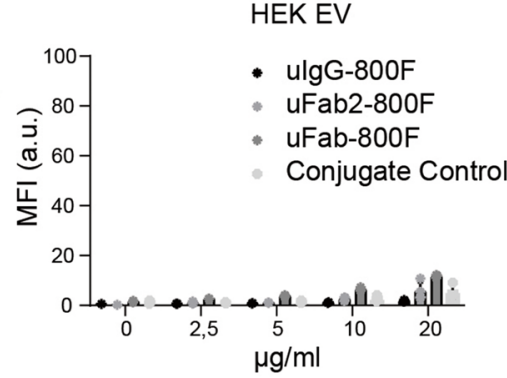

Fig. 1 In vitro validation of proteolytic cleavage and fluorescent conjugation of ulgG, uFab2, and uFab: a fluorescent signal on SDSPAGE gel at respectively $150-155 \mathrm{kDa}, 100-110 \mathrm{kDa}, 50-55 \mathrm{kDa}$, and $<10 \mathrm{kDa}$ for ulgG-800F, uFab2-800F, uFab-800F, and 800F. The latter represents free dye, indicating the purity of the conjugations in the other lanes. $\mathbf{b}$ Flow cytometry histograms demonstrating retained binding of uFab2 and uFab to wildtype uPAR (left) and the D2-D3 isotype (middle) after proteolytic cleavage of ulgG into the antibody fragments. Specificity is furthermore confirmed using empty vector transfected HEK cells (right) and a conjugate control as controls. c Cell-based plate assays showing dose-dependent binding of ulgG-800F, uFab2-800F, and uFab-800F and conjugate control to uPAR-positive HEK WT cells (left), uPAR D2-3 isotype-positive HEK cells (middle), and uPAR-negative EV cells (right). a.u., arbitrary units; MFI, mean fluorescent intensity.

Table 1 Comparison of $\mathrm{uIgG}, \mathrm{uFab} 2$, and $\mathrm{uFab}$ characteristics

\begin{tabular}{lllllllll}
\hline Tracer & $\begin{array}{l}\text { Targeting } \\
\text { domain }(\mathrm{Aa} \\
\mathrm{uPAR})\end{array}$ & $\mathrm{MW}(\mathrm{kDa})$ & Valency & $\mathrm{K}_{\mathrm{D}}(\mathrm{M})$ & Label & $\begin{array}{l}\text { Degree of } \\
\text { labeling }\end{array}$ & $\mathrm{K}_{\mathrm{D}}$ after conjugation $(\mathrm{M})$ & $\begin{array}{l}\text { Pre-/post-labeling } \\
\mathrm{K}_{\mathrm{D}} \text { difference }(T \\
\text { test; } p \text { ) }\end{array}$ \\
\hline $\mathrm{uIgG}$ & $268-275$ & $150-155$ & 2 & $2.19 \times 10^{-10} \pm 2.41^{-11}$ & $800 \mathrm{~F}$ & 0.9 & $2.56 \times 10^{-10} \pm 4.71^{-11}$ & 0.47 \\
$\mathrm{uFab} 2$ & $268-275$ & $100-110$ & 2 & $5.61 \times 10^{-10} \pm 1.61^{-10}$ & $800 \mathrm{~F}$ & 0.9 & $3.84 \times 10^{-10} \pm 1.51^{-10}$ & 0.53 \\
$\mathrm{uFab}$ & $268-275$ & $50-55$ & 1 & $8.66 \times 10^{-10} \pm 4.17^{-10}$ & $800 \mathrm{~F}$ & 1.2 & $1.43 \times 10^{-9} \pm 2.66^{-10}$ & 0.32 \\
\hline
\end{tabular}


increase of the MFI on HEK UPAR WT cells and the HEK uPAR D2-3 cells, but not on HEK EV control cells (Fig. 1c).

\section{Serial Imaging of Subcutaneous HT-29 Tumors with uIgG-800F, uFab2-800F, and uFab-800F}

Imaging of subcutaneous HT-29 tumors in mice demonstrated tumor specificity and identified the imaging window of 1-nmol uIgG-800F, 1-nmol uFab2-800F, and 2-nmol uFab$800 \mathrm{~F}$. Tumor accumulation of the full-sized antibody uIgG$800 \mathrm{~F}$ could be seen as early as $1 \mathrm{~h}$ after administration, but TBRs did not surpass two, an arbitrary cut-off point deemed suitable for NIR imaging, until $72 \mathrm{~h}$, due to relatively high background fluorescence at earlier time points [28]. The antibody fragments uFab2-800F and uFab-800F accumulated more rapidly in tumors, with TBRs surpassing two after $24 \mathrm{~h}$ (Fig. 2a; for serial images, see Suppl. Figure 2, see ESM).

Tumor fluorescence consistently remained above 0.1 arbitrary units (a.u.) with $\mathrm{uIgG}-800 \mathrm{~F}$, but dipped below it after $72 \mathrm{~h}$ with $\mathrm{uFab} 2-800 \mathrm{~F}$ and $36 \mathrm{~h}$ with Fab-800F (MFI uIgG-800F $120 \mathrm{~h}=0.156 \pm 0.016 \mathrm{au}$; MFI uFab2-800F $72 \mathrm{~h}=0.103 \pm 0.017 \mathrm{au} ;$ MFI uFab-800F $36 \mathrm{~h}=0.213 \pm 0.050$ au; Fig. 2a). During the imaging windows $72-120$ h, 24-72 h, and $24-36 \mathrm{~h}$, subcutaneous tumors were clearly identifiable with the clinical Artemis camera after uIgG-800F, uFab2800F, or uFab-800F administration, respectively (Fig. 2b). Extra-tumoral uIgG-800F accumulation was not visible. At all time points, kidney fluorescence could be seen through the skin after $\mathrm{uFAb} 2-800 \mathrm{~F}$ and $\mathrm{UFab}-800 \mathrm{~F}$ injection with kidney fluorescence surpassing tumor fluorescence (Fig. 2b-c).

\section{Fluorescence Imaging of Orthotopic Tumor Models with uIgG-800F, uFab2-800F, and uFab-800F}

Using three orthotopic cancer models, BxPC-3 PDAC, OSC19 tongue SCC, and HT-29 CRC peritoneal carcinomatosis, FGS potential of uIgG-800F, uFab2-800F, and uFab$800 \mathrm{~F}$ was compared. MFI of $1-\mathrm{nmol} \mathrm{uIgG}-800 \mathrm{~F}$, $1-\mathrm{nmol}$ uFab2-800F, and 2-nmol uFab-800F in $100 \mu \mathrm{L}$ solution was $2.08 \pm 0.38,2.47 \pm 0.44$, and $4.1 \pm 0.77$ au, respectively. Tumor burdens, measured by bioluminescence, did not differ significantly between tracers at the time of injection $(p=0.72$ for BxPC-3-luc2; $p=0.72$ for OSC-19-luc2-GFP; $p=0.31$ for HT29-luc2; Suppl. Figure 3, see ESM). Tumors were imaged at $96 \mathrm{~h}$ for $\mathrm{uIgG}-800 \mathrm{~F}, 48 \mathrm{~h}$ for $\mathrm{uFab} 2-800 \mathrm{~F}$, and $36 \mathrm{~h}$ for Fab$800 \mathrm{~F}$ after tracer administration.

Fluorescence clearly accumulated in abdominal PDAC BxPC-3-luc2 tumors after intravenous injection of the various tracers. In particular with uFab-800F, transcutaneous kidney and liver fluorescence interfered with optimal tumor imaging (Fig. 3a). TBRs were $2.5 \pm 0.4$ for $\mathrm{uIgG}-800 \mathrm{~F}, 3.3 \pm 1.2$ for uFab2-800F, and $2.3 \pm 1.0$ for uFab-800F. TBRs did not differ between all 3 tracers ( $p=0.58$; Fig. $3 b)$. Tumor MFI did not differ significantly but tumors could be visualized with shorter exposure times after $\mathrm{UIgG}-800 \mathrm{~F}$ injection as opposed to $\mathrm{uFab} 2-800 \mathrm{~F}$ or $\mathrm{uFab}-800 \mathrm{~F}$ ( $p=0.32$; multiple comparisons, uIgG- $800 \mathrm{~F}$ vs. uFab2-800F $p=0.32$, uIgG-800F vs. uFab-800F $p=0.46$, uFab2-800F vs. uFab-800F $p=0.95$; Fig. 3c).

After intravenous injection of uIgG-800F, uFab2-800F, or uFab-800F OSC-19-luc2-GFP, superficial squamous tongue tumors were easily identified with the Artemis clinical system. Shorter exposure times were needed after injection of uIgG-800F as opposed to uFab2-800F or uFab$800 \mathrm{~F}$ (Fig. 4a). TBRs of $2.8 \pm 0.5,3.6 \pm 0.6$ and $3.2 \pm 0.4$ were achieved for uIgG-800F, uFab2-800F, and uFab-800F, respectively. TBRs did not differ significantly between tracers $(p=0.33$; Fig. 4 b). MFI, however, did differ significantly with uIgG-800F having superior absolute fluorescence ( $p=0.01$; multiple comparisons, uIgG-800F vs. uFab2-800F $p=0.02$, uIgG-800F vs. uFab-800F $p=0.03$, uFab2-800F vs. uFab$800 \mathrm{~F} p=0.88$; Fig. $4 \mathrm{c}$ ).

Fluorescence detection of small lesions was studied using a HT-29 CRC peritoneal carcinomatosis model. Average lesion diameters were $3.2 \pm 0.8 \mathrm{~mm}, 3.7 \pm 1.2 \mathrm{~mm}$, and $4.7 \pm 0.6 \mathrm{~mm}$ for $\mathrm{uIgG}-800 \mathrm{~F}$, uFab2-800F, or uFab-800F $(p=0.18)$. Similar to the OSC-19-luc2-GFP tongue tumors and the BxPc-3-luc2 PDAC tumors, FGS with the Artemis clinical system was performed with lower exposure times in the UIgG-800F groups compared to the other two groups (Fig. 5a). TBRs, after uIgG-800F, uFab2-800F, or uFab$800 \mathrm{~F}$ administration, were $5.8 \pm 2.5,4.9 \pm 1.1$, and $5.4 \pm 0.8$, respectively, and did not differ significantly between tracer groups ( $p=0.81$; Fig. 5 b). Lesion MFI approached statistically significant difference with $\mathrm{IgG}-800 \mathrm{~F}$ being superior ( $p=0.07$; multiple comparisons, uIgG- $800 \mathrm{~F}$ vs. uFab2-800F $p=0.09, \mathrm{uIgG}-800 \mathrm{~F}$ vs. uFab-800F $p=0.14, \mathrm{uFab} 2-800 \mathrm{~F}$ vs. uFab-800F $p=0.99$; Fig. 5c).

\section{Ex Vivo Characterization of Normal and Tumor Tissue}

Ex vivo macroscopic analysis of normal tissue showed that most of the fluorescence was located in the metabolizing organs (Fig. 6a-b). Liver MFI differed significantly between tracers with $\mathrm{uFab}-800 \mathrm{~F}$ having the highest, and $\mathrm{uIgG}-800 \mathrm{~F}$ and $\mathrm{uFab} 2-800 \mathrm{~F}$ having similar MFI's $(p<0.01$; multiple comparisons, uIgG-800F vs. uFab2-800F $p=0.28$, uIgG$800 \mathrm{~F}$ vs. uFab-800F $p<0.01$, uFab2-800F vs. uFab-800F $p<0.01)$. Kidney MFI differed significantly between all tracer types with uIgG-800F having the lowest MFI $(p<0.01$; multiple comparisons, uIgG-800F vs. uFab2-800F $p<0.01$, uIgG-800F vs. uFab-800F $p<0.01$, uFab2-800F vs. uFab$800 \mathrm{~F} p<0.01$ ). MFI of the other organs did not approach or pass that of tumor MFI's and did not significantly impact background fluorescence. Ex vivo macroscopic tumor fluorescence could be clearly visualized with all three tracers. uIgG-800F tumor MFI, however, was consistently higher than uFab2-800F or uFab-800F in all three orthotopic tumor models (Fig. 6b). Post-mortem overlay of histology with 
a
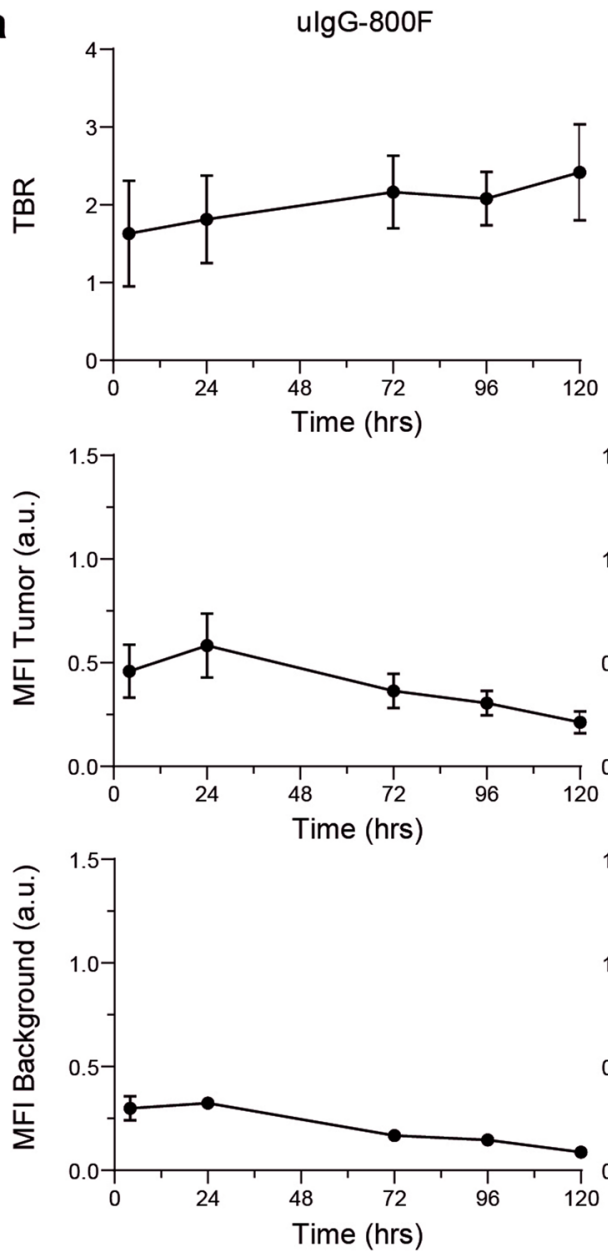

b
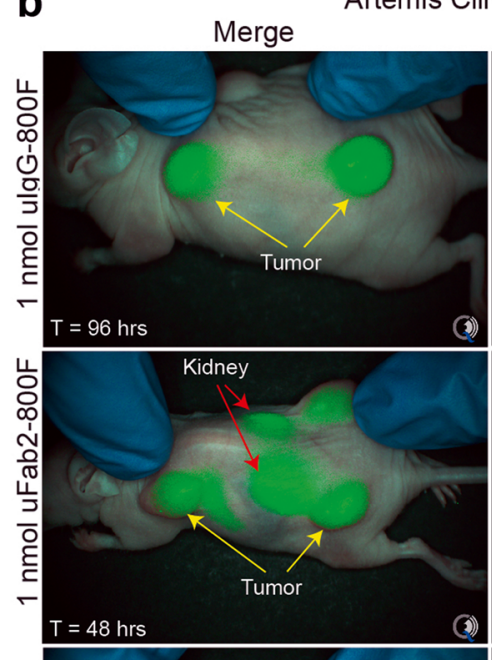

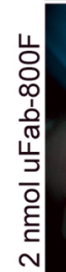

Kidney
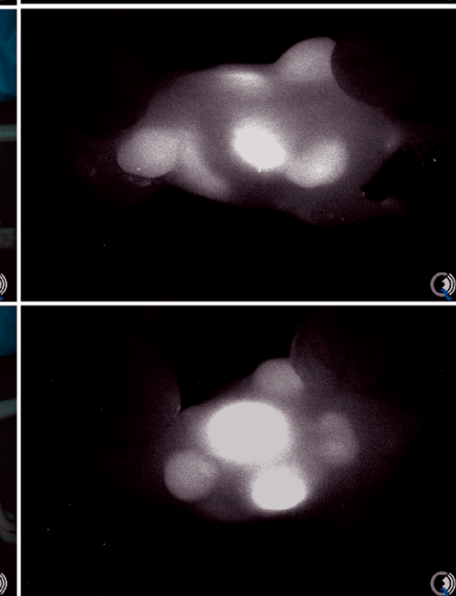
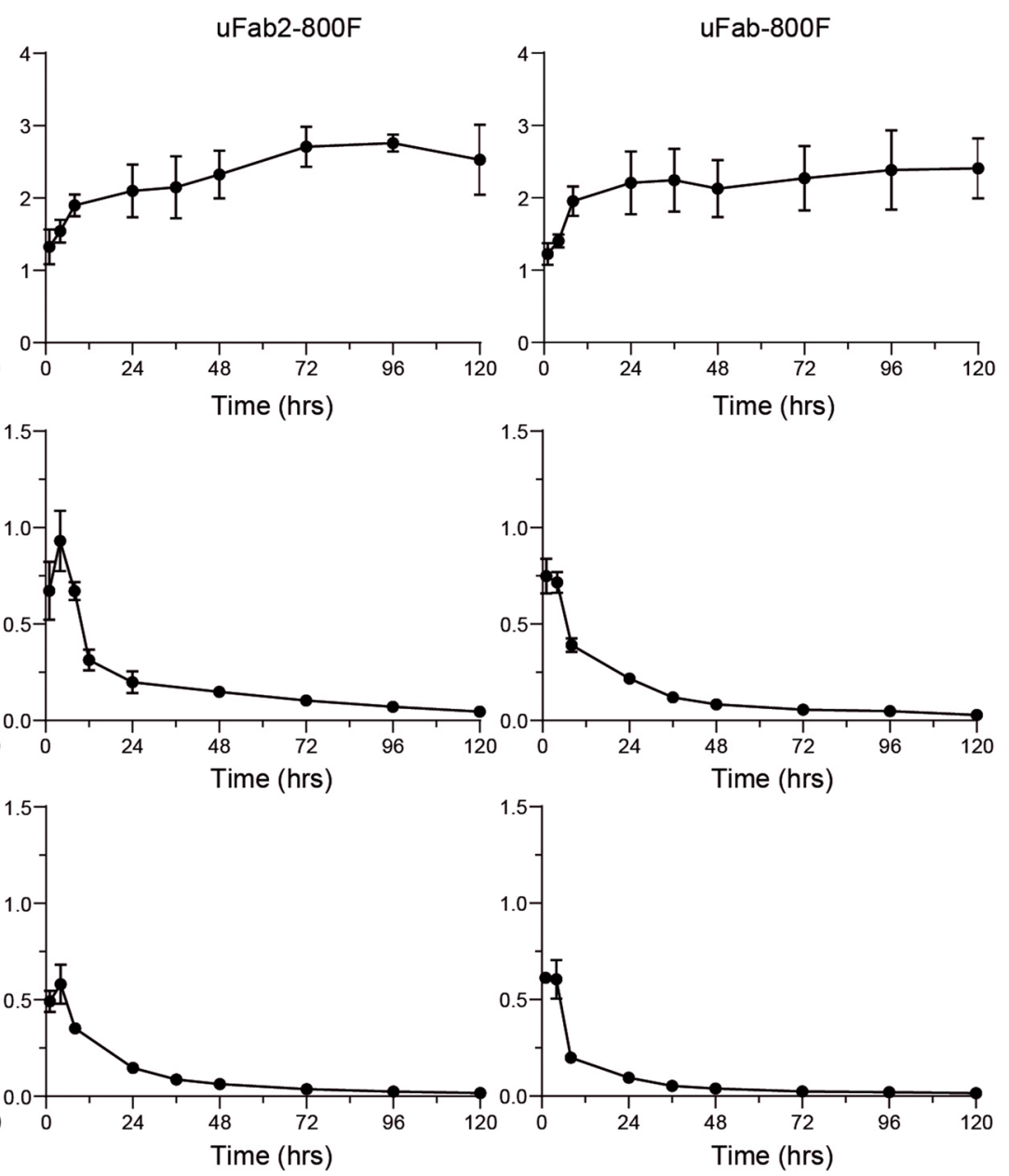

$800 \mathrm{~nm}$

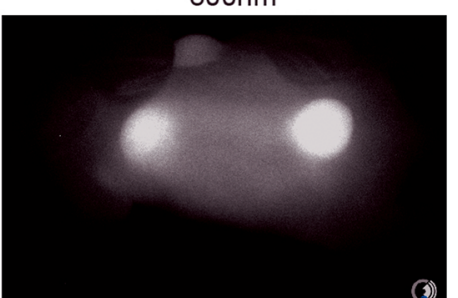

C
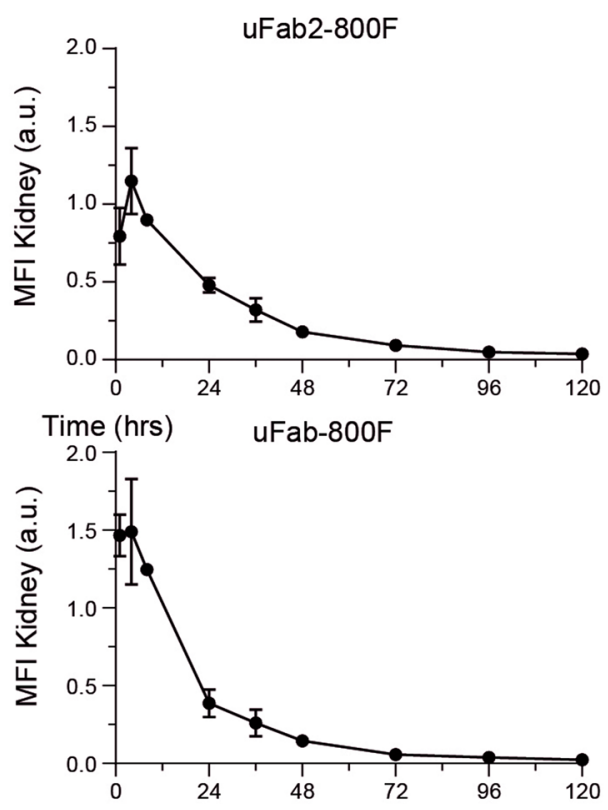
4 Fig. 2 In vivo serial imaging of ulgG-800F, uFab2-800F, and uFab800F in subcutaneous HT-29 tumor bearing mice: a TBR and MFI measured using the Pearl over time after injection of 1-nmol ulgG-800F, 1-nmol uFab2-800F, and 2-nmol uFab-800F. b Merge and $800-\mathrm{nm}$ images taken with the Artemis clinical camera demonstrating real-time tumor imaging at $96 \mathrm{~h}$ for ulgG-800F, $48 \mathrm{~h}$ for uFab2-800F, and $36 \mathrm{~h}$ for uFab-800F. These time slots are shown as they fall within the optimal imaging window for each of the tracers. For images at other time periods, see Suppl. Figure 2 (ESM). Tumor and kidney fluorescence are identified with yellow and red arrows, respectively. c Transcutaneous kidney fluorescence over time after uFab2-800F and uFab-800F administration. a.u., arbitrary units; hrs, hours; MFI, mean fluorescence intensity; TBR, tumor-to-background ratio; T, time.

fluorescent signal confirmed tumor-specific accumulation at the tumor cells using all three tracers (Fig. 6c).

\section{Discussion}

Optimizing NIR contrast agents for molecular imaging is a challenge, as tumor visualization is dependent on a multitude of factors including, but not limited to, tracer size, valency, affinity, and labeling ratio [29-31]. By decreasing size, the current study evaluated whether tumor-specific imaging could be retained and concurrently pharmacokinetics improved for the validated humanized anti-uPAR monoclonal antibody MNPR-101. Although the 100-kDa F(ab') 2 and 50-kDa Fab performed similarly in vitro to the $150 \mathrm{kDa} \mathrm{IgG}$, in vivo imaging resulted in more rapid FGS with the two smaller fragments at the cost of peak tumor fluorescence.

Only few reports have directly compared antibodies with their fragments for FGS. Fluorescent anti-HER3 IgG and single-chain variable fragment ( $\mathrm{scFv})-\mathrm{Fc}$ had higher tumor

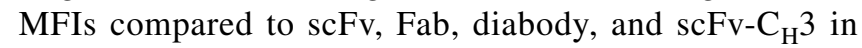
HNSCC xenografts [32]. In another study, EphB4-targeting IgG showed maximum tumor uptake while $\mathrm{F}\left(\mathrm{ab}{ }^{\prime}\right)_{2}$ and Fab resulted in step-wise lower tumor signal [33]. In a third study, using ICAM-1 Fabs, tumor MFI decreased to such an extent compared to the full-sized antibody that it matched that of non-specific controls [34]. These results, although differing in constructs and/or targets, match ours and are comparable to previous PET studies where CD105 targeting $\mathrm{F}\left(\mathrm{ab}{ }^{\prime}\right)_{2}$ and Fab lead to earlier imaging but lower peak signal [22, 23].

Previously, however, we achieved high peak tumor signal with the epithelial cell adhesion molecule (EpCAM) targeting Fab VB6-845-IRDye800CW resulting in clinical translation (NTR7570) [35]. Expression characteristics of the targeted receptor could explain this difference in peak MFI attained by the Fabs. The colorectal HT-29 and breast MCF-7 cell lines used by Boogerd et al. express respectively 195,000 and 260,000 copies of EpCAM while BxPc-3, OSC-19, and HT-29 have 10,000-30,000 copies of uPAR [14, 35]. For receptors with lower cell expression, like UPAR, HER3, and EphB4, longer circulation times could be required to establish sufficient accumulation of tracer in tumors and clearance from background tissue [32, 36, 37].

In addition to receptor expression, tracer extravasation and clearance can clarify differences in tumor uptake. IgGs compensate their unfavorable slow extravasation characteristics with extended circulation times, in part due to their Fc receptor-mediated recycling and minimal renal clearance, providing a tracer multiple chances for extravasation [17, 38]. In contrast, the improved extravasation of molecules ranging in size between 20 and $50 \mathrm{kDa}$ is not in proportion to the rapid renal clearance resulting in worsened tumor accumulation compared to IgGs [39, 40]. At and below $20 \mathrm{kDa}$, like for nanobodies and peptides, renal clearance is practically at first pass and as such cannot improve, while extravasation increases rapidly with decreasing size resulting in favorable tumor accumulation again [40]. Once in the tumor compartment, the superior diffusion of smaller molecules will result in a more homogenous tumor distribution compared to their larger counterparts [31].

Nonetheless, these disadvantages can be compensated by improving target affinity as there is an inverse relation between size and affinity $\left(\mathrm{K}_{\mathrm{D}}\right)$ required to reach similar tumor uptakes. $\mathrm{K}_{\mathrm{D}} \mathrm{s}$ of $10^{-8}$ to $10^{-6} \mathrm{M}$ for antibodies result in similar degrees of tumor retention as Fabs or scFvs with a $\mathrm{K}_{\mathrm{D}}$ of $10^{-10}$ to $10^{-8} \mathrm{M}$ [41-43]. Our anti-uPAR Fab has a nanomolar affinity, approximately ten-fold higher than the ICAM-1 Fab, and similar to the HER-3 Fab while VB6-845-IRDye800CW has a picomolar affinity [35]. In conclusion, the combination of receptor density and tracer affinity in combination with size plays a pivotal role in achieving high peak intensity, possibly explaining why VB6-845-IRDye800CW achieved superior imaging conditions and why, for the other targets, the larger agents resulted in superior MFIs.

The currently described uPAR-targeting antibody fragments are not the only uPAR-targeting contrast agents under development. The growth factor domain of urokinase (ATF), the natural ligand of uPAR with a $\mathrm{K}_{\mathrm{D}}$ of $2.5 \times 10^{-10} \mathrm{M}$, has been coupled with various dyes like Cy5.5 and NIR830 for FGS as approximately $18-\mathrm{kDa}$ peptides and much larger nanoparticle probes [44-46]. The 9-mer peptide ICG-GluGlu-AE105 also targets the uPAR-ATF interaction with a $\mathrm{K}_{\mathrm{D}}$ of $134 \times 10^{-9} \mathrm{M}$ and results in rapid (6-24 h) tumor localization in various in vivo xenograft models [47-49]. While the delayed imaging window of $\mathrm{uIgG}-800 \mathrm{~F}$ and rapid imaging window of ATF peptides and ICG-Glu-Glu-AE105 are evident, peak fluorescence is much harder to compare as these constructs not only differ in size and affinity but also in fluorophore. In this case, IRDye800CW has a higher extinction coefficient and quantum yield than ICG and can be expected to be brighter [50]. Lastly, the targeting epitope is a crucial difference between ATF peptides, ICG-Glu-Glu-AE105, and huIgG-800CW where the former two, as uPA competitors, are dependent on low endogenous uPA expression and the latter is not [51]. Co-injection experiments of uPA and ICGGlu-Glu-AE105 have resulted in an almost 50\% decrease in fluorescence signal [47]. huIgG-800CW specifically targets 

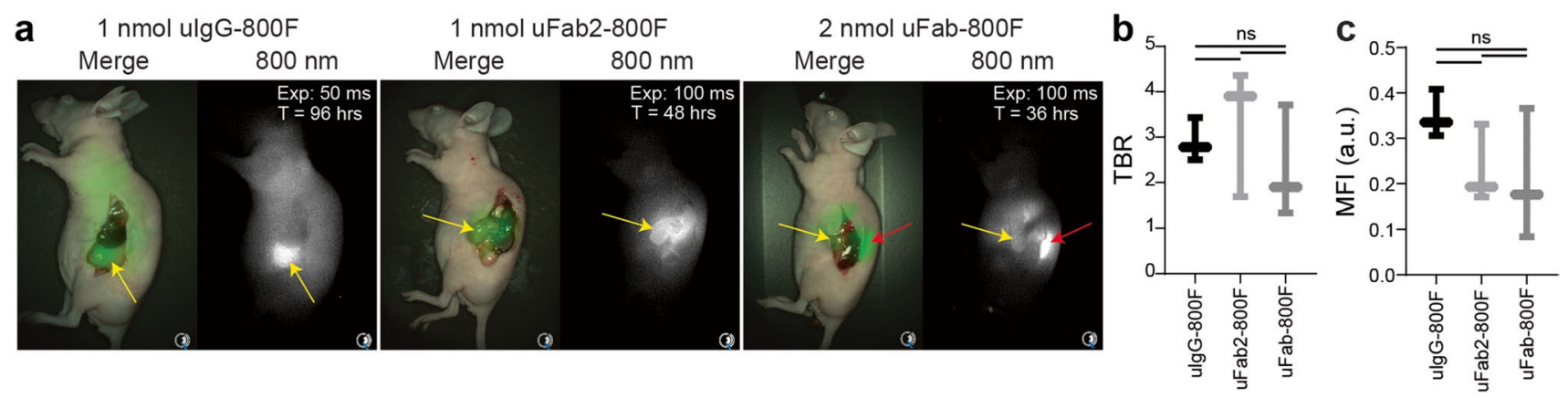

Fig. 3 In vivo fluorescence imaging of orthotopic BxPC-3 pancreas adenocarcinomas with ulgG-800F, uFab2-800F, and uFab-800F during the optimal time period: a NIR images of orthotopic BxPC-3 pancreas adenocarcinomas taken with the Artemis clinical camera $96 \mathrm{~h}$ are ulgG-800F (left panel), $48 \mathrm{~h}$ are uFab2-800F (middle panel), and $36 \mathrm{~h}$ after uFab-800F (left panel) administration. Tumors are identified with a yellow arrow and kidney fluorescence, when present, with a red arrow. Note the lower exposure time needed after ulgG-800F administration reflecting a higher MFI. b TBRs and c tumor MFIs measured using the Pearl do not differ significantly between ulgG-800F, uFab2-800F, and uFab-800F at respectively 96 h, 48 h,and 36 h after injection. a.u., arbitrary units; hrs, hours; MFI, mean fluorescence intensity; ns, not significant; TBR, tumor-to-background ratio; T, time.
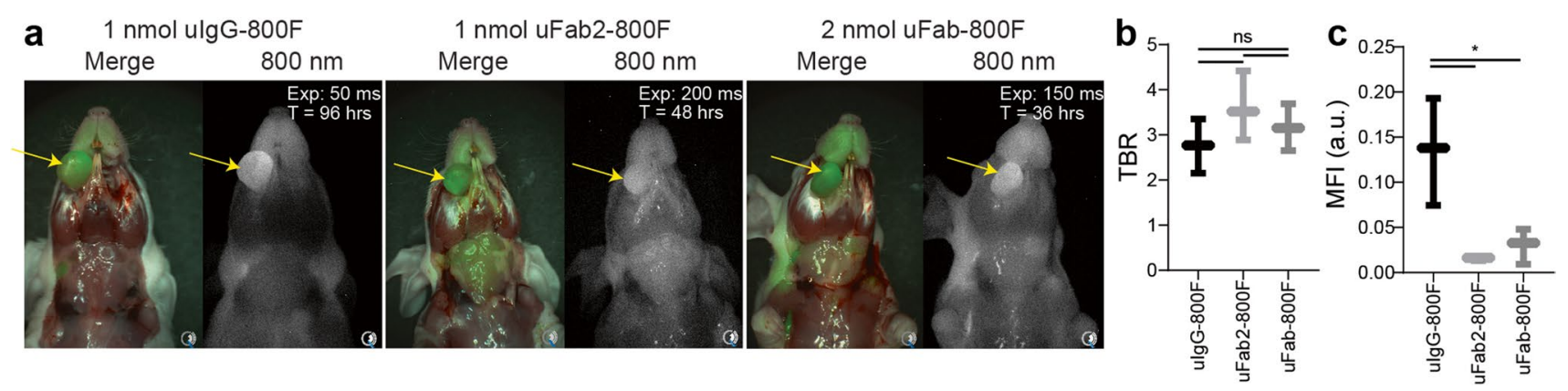

Fig. 4 In vivo fluorescence imaging of ulgG-800F, uFab2-800F, and uFab-800F during the optimal imaging window in orthotopic squamous cell OSC-19 tongue tumors: a NIR images taken with the Artemis clinical camera of orthotopic OSC-19 tongue tumors $96 \mathrm{~h}$ after ulgG-800F, $48 \mathrm{~h}$ after uFab2-800F, and $36 \mathrm{~h}$ after uFab-800F administration. Tumors are identified with a yellow arrow. Note the lower exposure time needed to visualize the tumor after ulgG-800F administration compared to uFab2-800F and uFab-800F. $\mathbf{b}$ TBRs and $\mathbf{c}$ tumor MFIs measured using the Pearl for ulgG-800F, uFab2-800F, and uFab-800F at respectively 96,48 , and $36 \mathrm{~h}$ after administration demonstrating similar TBRs between the groups but a higher MFI for ulgG-800F. a.u., arbitrary units; hrs, hours; MFI, mean fluorescence intensity; NIR, near-infrared; ns, not significant; TBR, tumor-to-background ratio; T, time.
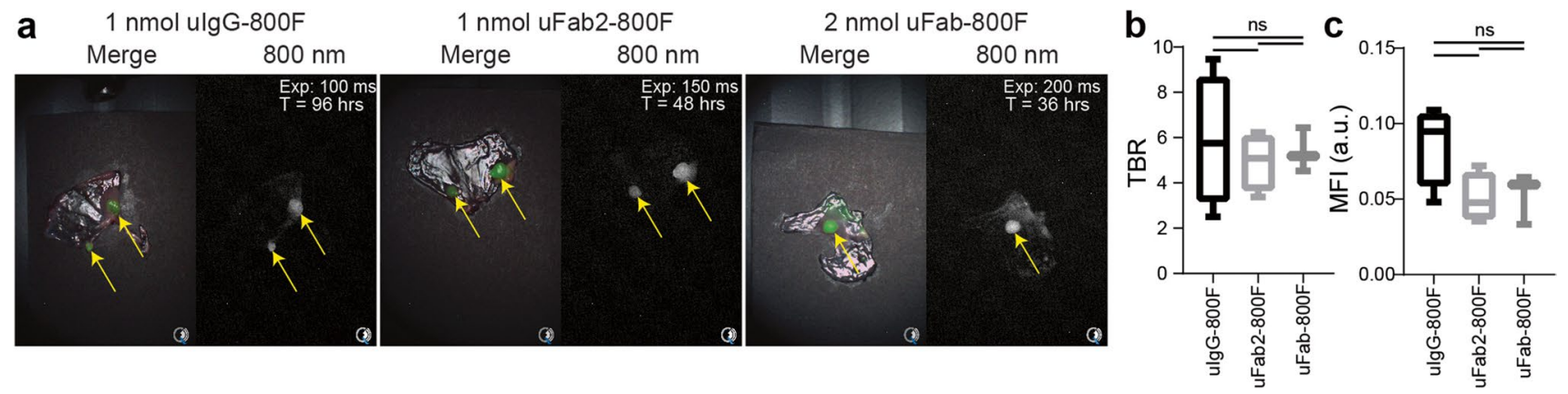

Fig. 5 In vivo fluorescence imaging of small lesions with ulgG-800F, uFab2-800F, and uFab-800F at the optimal imaging window using an orthotopic HT-29 colorectal cancer peritoneal carcinomatosis model: a NIR images taken with the Artemis clinical camera of the peritoneum containing HT-29 lesions $96 \mathrm{~h}$ are ulgG-800F, $48 \mathrm{~h}$ after uFab2-800F, and $36 \mathrm{~h}$ after uFab-800F administration. Tumors are identified with the yellow arrow. Note the differing exposure times needed to create similar images reflecting the differing MFI achieved with each of the tracers. b Average TBR and c lesion MFI measured using the Pearl achieved $96 \mathrm{~h}$ after ulgG-800F, $48 \mathrm{~h}$ after uFab2-800F, and $36 \mathrm{~h}$ after uFab-800F injection. a.u., arbitrary units; hrs, hours; MFI, mean fluorescence intensity; NIR, near-infrared; ns, not significant; TBR, tumor-to-background ratio; T, time. 
a

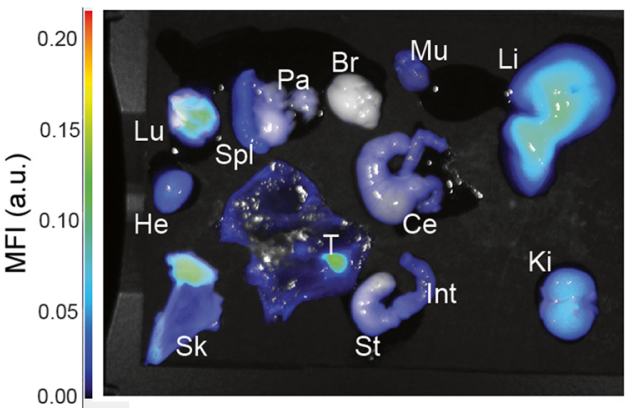

b

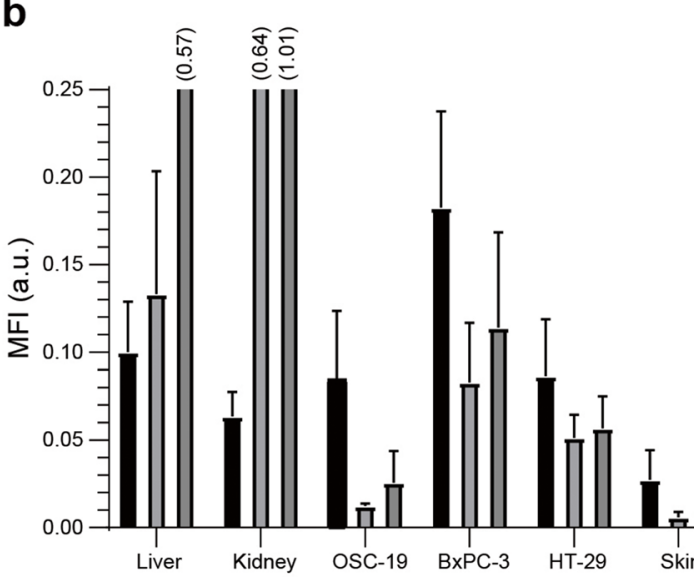

$1 \mathrm{nmol}$ uFab2-800F, 48 hrs

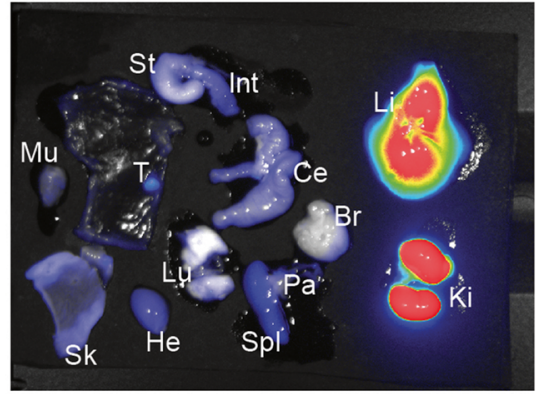

2 nmol uFab-800F, 36 hrs

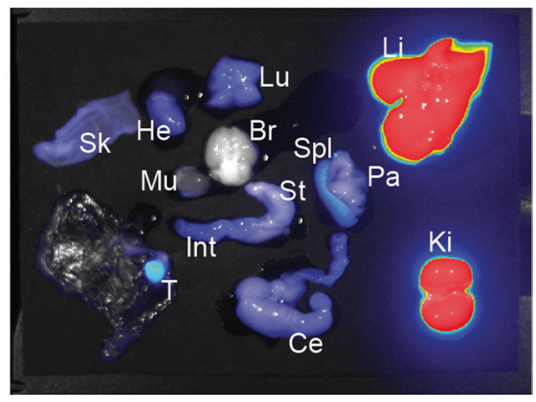

C

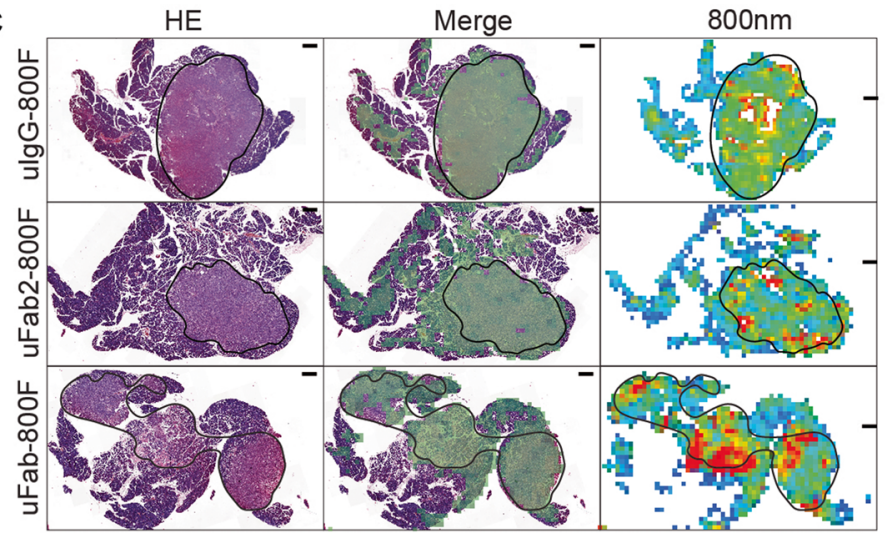

Fig. 6 Ex vivo macroscopic and microscopic fluorescence biodistribution of ulgG-800F, uFab2-800F, and uFab-800F at optimal imaging window: a ex vivo biodistribution visualized with the Pearl and $\mathbf{b}$ quantified in a bar graph of ulgG-800F, uFab2-800F, and Fab-800F at respectively 96,48 , and $36 \mathrm{~h}$ after administration. Fluorescence intensities across the images are matched. (c) Overlay of HE staining and 800-nm fluorescence showing fluorescence accumulation in tumor area compared to surrounding normal tissue. Tumor tissue is delineated by the black line. The black line represents $500 \mu \mathrm{m}$. a.u., arbitrary units; $\mathrm{Br}$, brain; Ce, cecum; He, heart; HE, hematoxylin and eosin; Hrs, hours; Int, intestine; Ki, kidneys; Li, liver; Lu, lung; MFI, mean fluorescence intensity; Mu, muscle; nm, nanometer; Pa, pancreas; Sk, skin; Spl, spleen; St, stomach.

the D2-D3 uPAR isotype, often found to be overexpressed in cancer $[9,16]$.

While the conclusions drawn in this study reflect the literature, the current study contains a couple limitations. Although the limited group sizes were sufficient according to previous sample size calculations to identify the most relevant differences in TBRs (increase in 50\%, see ESM), more subtle differences could have been missed. Ethical constraints, however, limited researching this avenue. In addition, using orthotopic models minimalized the time points tumors could be visualized in vivo, potentially missing better imaging moments. This was negated by first imaging the tracers serially in a subcutaneous tumor model and carefully defining what determined a suitable time window (see ESM). Lastly, administrating tracers based on fluorescence as opposed to antigen binding sites could potentially have allowed for a more accurate comparison of fluorescence intensity; however, the results, if different at all when injecting a surplus 
amount of tracer, would have skewed the conclusion even more towards full-sized antibodies.

\section{Conclusions}

To conclude, this study successfully introduces two novel UPAR antibody-fragment tracers based on the extensively validated MNPR-101 humanized parental antibody. $\left(\mathrm{F}(\mathrm{ab})^{\prime}\right)_{2}$ and $\mathrm{Fab}$ greatly improved time-to-imaging while the whole antibody demonstrated superior peak fluorescence. In the clinic, the various pharmacokinetic profiles of the tracers should be considered as Fab utilization is better in (semi-) acute settings (same-day or next-day surgery), but should not be used when absolute receptor expression is expected to be relatively low. In these cases, surgeons should veer towards full-sized antibodies or constructs smaller than $20 \mathrm{kDa}$, such as nanobodies or peptides.

Supplementary Information The online version contains supplementary material available at https://doi.org/10.1007/s11307-021-01657-2.

Acknowledgements The authors would like to acknowledge N.G. DekkerEnsink and R.L.P Vlierberghe for their excellent technical assistance. Dr. Sier was in part funded by the European Commission under two Marie Skłodowska-Curie Action awards: H2020-MSCA-RISE-2019 (project number: 872860-PRISAR2) and H2020-WIDESPREAD-2018-Twinning (project number: 852985-SIMICA).

Author Contribution The manuscript has been seen and approved by all authors. VB, PK, AM, AV, and CS were responsible for conception and design. VB, LvM, SB, LI, HdJ, CS, FV, and RC acquired data. VB, LvM, LI, HdJ, and CS analyzed and interpreted data. VB, LvM, and CS drafted the manuscript and performed the statistical analysis. MR performed important administrative, technical, or material support. PK, AV, and CS supervised the project. All authors critically revised the manuscript for important intellectual content.

\section{Declarations}

Statement of Human and Animal Rights This article does not contain any studies with human participants.

All animal experiments were approved by the Dutch Central Commission for Animal Experimentation (AVD1160020172925). Experiments were performed in accordance with the code of practice "Dierproeven In Het Kankeronderzoek." All applicable institutional and/or national guidelines for the care and use of animals were followed.

Conflict of Interest Dr. Iamele, Dr. de Jonge, and Dr Scotti report non-financial support from Ardis S.r.l., during the conduct of the study. Dr. Mazar reports personal fees and other from Monopar Therapeutics, outside the submitted work. In addition, Dr. Mazar has a patent US 8105602; US 8101726 issued. All other authors have nothing to disclose.

Open Access This article is licensed under a Creative Commons Attribution 4.0 International License, which permits use, sharing, adaptation, distribution and reproduction in any medium or format, as long as you give appropriate credit to the original author(s) and the source, provide a link to the Creative Commons licence, and indicate if changes were made. The images or other third party material in this article are included in the article's Creative Commons licence, unless indicated otherwise in a credit line to the material. If material is not included in the article's Creative Commons licence and your intended use is not permitted by statutory regulation or exceeds the permitted use, you will need to obtain permission directly from the copyright holder. To view a copy of this licence, visit http://creativeco mmons.org/licenses/by/4.0/

\section{References}

1. Allemani C, Matsuda T, Di Carlo V et al (2018) Global surveillance of trends in cancer survival 2000-14 (CONCORD-3): analysis of individual records for 37513025 patients diagnosed with one of 18 cancers from 322 population-based registries in 71 countries. Lancet (London, England) 391:1023-1075

2. Miller KD, Nogueira L, Mariotto AB et al (2019) Cancer treatment and survivorship statistics, 2019. CA Cancer J Clin 69:363-385

3. Khan MA, Hakeem AR, Scott N, Saunders RN (2015) Significance of $\mathrm{R} 1$ resection margin in colon cancer resections in the modern era. Colorectal disease: the official journal of the Association of Coloproctology of Great Britain and Ireland 17:943-953

4. Ghaneh P, Kleeff J, Halloran CM et al (2019) The impact of positive resection margins on survival and recurrence following resection and adjuvant chemotherapy for pancreatic ductal adenocarcinoma. Ann Surg 269:520-529

5. Wolf AS, Swanson SJ, Yip R et al (2017) The impact of margins on outcomes after wedge resection for stage I non-small cell lung cancer. Ann Thorac Surg 104:1171-1178

6. Hernot S, van Manen L, Debie P, Mieog JSD, Vahrmeijer AL (2019) Latest developments in molecular tracers for fluorescence image-guided cancer surgery. Lancet Oncol 20:e354-e367

7. van Manen L, Handgraaf HJM, Diana M et al (2018) A practical guide for the use of indocyanine green and methylene blue in fluorescenceguided abdominal surgery. J Surg Oncol 118:283-300

8. Boonstra MC, Verspaget HW, Ganesh S et al (2011) Clinical applications of the urokinase receptor (uPAR) for cancer patients. Curr Pharm Des 17:1890-1910

9. Baart VM, Houvast RD, de Geus-Oei LF et al (2020) Molecular imaging of the urokinase plasminogen activator receptor: opportunities beyond cancer. EJNMMI Res 10:87

10. Baart VM, van Duijn C, van Egmond SL, et al. (2020) EGFR and $\alpha v \beta 6$ as promising targets for molecular imaging of cutaneous and mucosal squamous cell carcinoma of the head and neck region. Cancers 12

11. de Geus SW, Baart VM, Boonstra MC et al (2017) Prognostic impact of urokinase plasminogen activator receptor expression in pancreatic cancer: malignant versus stromal cells. Biomarker insights 12:1177271917715443

12. Boonstra MC, Verbeek FP, Mazar AP et al (2014) Expression of uPAR in tumor-associated stromal cells is associated with colorectal cancer patient prognosis: a TMA study. BMC Cancer 14:269

13. Fosbøl M, Kurbegovic S, Johannesen HH et al (2021) Urokinase-type plasminogen activator receptor (uPAR) PET/MRI of prostate cancer for noninvasive evaluation of aggressiveness: comparison with Gleason score in a prospective phase 2 clinical trial. Journal of nuclear medicine : official publication, Society of Nuclear Medicine 62:354-359

14. Boonstra MC, van Driel PB, van Willigen DM et al (2015) uPARtargeted multimodal tracer for pre- and intraoperative imaging in cancer surgery. Oncotarget 6:14260-14273

15. Boonstra MC, Van Driel P, Keereweer S et al (2017) Preclinical uPARtargeted multimodal imaging of locoregional oral cancer. Oral Oncol $66: 1-8$

16. Baart VM, van der Horst G, Deken MM et al (2021) A multimodal molecular imaging approach targeting urokinase plasminogen activator receptor for the diagnosis, resection and surveillance of urothelial cell carcinoma. Eur J Cancer 146:11-20

17. Zalevsky J, Chamberlain AK, Horton HM et al (2010) Enhanced antibody half-life improves in vivo activity. Nat Biotechnol 28:157-159

18. Holliger P, Hudson PJ (2005) Engineered antibody fragments and the rise of single domains. Nat Biotechnol 23:1126-1136

19. de Valk KS, Deken MM, Schaap DP et al (2021) Dose-finding study of a CEA-targeting agent, SGM-101, for intraoperative fluorescence imaging of colorectal cancer. Ann Surg Oncol 28:1832-1844

20. Williams LE, Wu AM, Yazaki PJ et al (2001) Numerical selection of optimal tumor imaging agents with application to engineered antibodies. Cancer Biother Radiopharm 16:25-35 
21. Debie P, Devoogdt N, Hernot S (2019) Targeted nanobody-based molecular tracers for nuclear imaging and image-guided surgery. Antibodies (Basel) 8 .

22. Hong H, Zhang Y, Orbay H et al (2013) Positron emission tomography imaging of tumor angiogenesis with a $(61 / 64) \mathrm{Cu}$-labeled $\mathrm{F}(\mathrm{ab}$ ') (2) antibody fragment. Mol Pharm 10:709-716

23. Zhang Y, Hong H, Orbay H, et al. (2013) PET imaging of CD105/ endoglin expression with a ${ }^{61} /{ }^{64} \mathrm{Cu}$-labeled $\mathrm{Fab}$ antibody fragment. Eur J Nucl Med Mol Imaging 40:759-767

24. van Driel PB, Boonstra MC, Prevoo HA et al (2016) EpCAM as multitumour target for near-infrared fluorescence guided surgery. BMC Cancer 16:884

25. Tseng W, Leong X, Engleman E (2007) Orthotopic mouse model of colorectal cancer. J Vis Exp:484

26. Kim MP, Evans DB, Wang H, Abbruzzese JL, Fleming JB, Gallick GE (2009) Generation of orthotopic and heterotopic human pancreatic cancer xenografts in immunodeficient mice. Nat Protoc 4:1670-1680

27. Schindelin J, Arganda-Carreras I, Frise E et al (2012) Fiji: an opensource platform for biological-image analysis. Nat Methods 9:676-682

28. Hoogstins CE, Weixler B, Boogerd LS, et al. (2017) In search for optimal targets for intraoperative fluorescence imaging of peritoneal metastasis from colorectal cancer. Biomarkers in cancer 9:1179299x17728254

29. Muchekehu R, Liu D, Horn M et al (2013) The effect of molecular weight, PK, and valency on tumor biodistribution and efficacy of antibody-based drugs. Transl Oncol 6:562-572

30. Vira S, Mekhedov E, Humphrey G, Blank PS (2010) Fluorescentlabeled antibodies: balancing functionality and degree of labeling. Anal Biochem 402:146-150

31. Xenaki KT, Oliveira S, van Bergen En, Henegouwen PMP (2017) Antibody or antibody fragments: implications for molecular imaging and targeted therapy of solid tumors. Front Immunol 8:1287

32. El-Sayed A, Bernhard W, Barreto K et al (2018) Evaluation of antibody fragment properties for near-infrared fluorescence imaging of HER3positive cancer xenografts. Theranostics 8:4856-4869

33. Li D, Liu S, Liu R et al (2013) EphB4-targeted imaging with antibody h131, h131-F(ab')2 and h131-Fab. Mol Pharm 10:4527-4533

34. Leelawattanachai J, Kwon KW, Michael P, Ting R, Kim JY, Jin MM (2015) Side-by-side comparison of commonly used biomolecules that differ in size and affinity on tumor uptake and internalization. PloS one 10:e 0124440

35. Boogerd LSF, Boonstra MC, Prevoo H et al (2019) Fluorescenceguided tumor detection with a novel anti-EpCAM targeted antibody fragment: preclinical validation. Surg Oncol 28:1-8

36. Rosestedt M, Andersson KG, Mitran B et al (2015) Affibody-mediated PET imaging of HER3 expression in malignant tumours. Sci Rep 5:15226

37. Stammes MA, Prevoo HA, Ter Horst MC, et al. (2017) Evaluation of EphA2 and EphB4 as targets for image-guided colorectal cancer surgery. Int J Mol Sci 18
38. Dreher MR, Liu W, Michelich CR, Dewhirst MW, Yuan F, Chilkoti A (2006) Tumor vascular permeability, accumulation, and penetration of macromolecular drug carriers. J Natl Cancer Inst 98:335-344

39. Thurber GM, Zajic SC, Wittrup KD (2007) Theoretic criteria for antibody penetration into solid tumors and micrometastases. Journal of nuclear medicine : official publication, Society of Nuclear Medicine 48:995-999

40. Wittrup KD, Thurber GM, Schmidt MM, Rhoden JJ (2012) Practical theoretic guidance for the design of tumor-targeting agents. Methods Enzymol 503:255-268

41. Schmidt MM, Wittrup KD (2009) A modeling analysis of the effects of molecular size and binding affinity on tumor targeting. Mol Cancer Ther 8:2861-2871

42. Fouliard S, Chenel M, Marcucci F (2013) Influence of the duration of intravenous drug administration on tumor uptake. Front Oncol 3:192

43. Adams GP, Schier R, Marshall K et al (1998) Increased affinity leads to improved selective tumor delivery of single-chain Fv antibodies. Cancer Res 58:485-490

44. Yang L, Sajja HK, Cao Z et al (2013) uPAR-targeted optical imaging contrasts as theranostic agents for tumor margin detection. Theranostics 4:106-118

45. Abdalla MO, Karna P, Sajja HK et al (2011) Enhanced noscapine delivery using uPAR-targeted optical-MR imaging trackable nanoparticles for prostate cancer therapy. Journal of controlled release : official journal of the Controlled Release Society 149:314-322

46. Lin L, Gårdsvoll H, Huai Q, Huang M, Ploug M (2010) Structure-based engineering of species selectivity in the interaction between urokinase and its receptor: implication for preclinical cancer therapy. J Biol Chem 285:10982-10992

47. Juhl K, Christensen A, Persson M, Ploug M, Kjaer A (2016) Peptidebased optical uPAR imaging for surgery: in vivo testing of ICG-GluGlu-AE105. PloS one 11:e0147428

48. Christensen A, Juhl K, Persson M et al (2017) uPAR-targeted optical near-infrared (NIR) fluorescence imaging and PET for image-guided surgery in head and neck cancer: proof-of-concept in orthotopic xenograft model. Oncotarget 8:15407-15419

49. Juhl K, Christensen A, Rubek N, Karnov KKS, von Buchwald C, Kjaer A (2019) Improved surgical resection of metastatic pancreatic cancer using UPAR targeted in vivo fluorescent guidance: comparison with traditional white light surgery. Oncotarget 10:6308-6316

50. Gioux S, Choi HS, Frangioni JV (2010) Image-guided surgery using invisible near-infrared light: fundamentals of clinical translation. Mol Imaging 9:237-255

51. Baart VM, Boonstra MC, Sier CFM (2017) uPAR directed-imaging of head-and-neck cancer. Oncotarget 8:20519-20520

Publisher's Note Springer Nature remains neutral with regard to jurisdictional claims in published maps and institutional affiliations. 